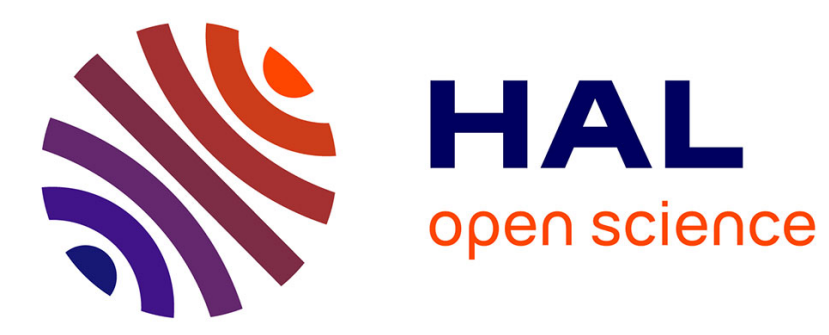

\title{
General tube law for collapsible thin and thick-wall tubes
}

\author{
Pavel Kozlovsky, Uri Zaretsky, Ariel Jaffa, David Elad
}

\section{To cite this version:}

Pavel Kozlovsky, Uri Zaretsky, Ariel Jaffa, David Elad. General tube law for collapsible thin and thick-wall tubes. Journal of Biomechanics, 2014, 10.1016/j.jbiomech.2014.04.033 . hal-01302932

\section{HAL Id: hal-01302932 \\ https://hal.science/hal-01302932}

Submitted on 15 Apr 2016

HAL is a multi-disciplinary open access archive for the deposit and dissemination of scientific research documents, whether they are published or not. The documents may come from teaching and research institutions in France or abroad, or from public or private research centers.
L'archive ouverte pluridisciplinaire HAL, est destinée au dépôt et à la diffusion de documents scientifiques de niveau recherche, publiés ou non, émanant des établissements d'enseignement et de recherche français ou étrangers, des laboratoires publics ou privés. 


\title{
General tube law for collapsible thin and thick-wall tubes
}

\author{
Pavel Kozlovsky, Uri Zaretsky, David Elad \\ Department of Biomedical Engineering, Faculty of Engineering, Tel Aviv University, Tel Aviv, Israel
}

\author{
Ariel J. Jaffa \\ Ultrasound Unit in Obstetrics and Gynecology, Lis Maternity Hospital, Tel Aviv Sourasky, Medical Center, Tel Aviv and Sackler Faculty of Medicine, \\ Tel Aviv University, Tel Aviv, Israel
}

\begin{abstract}
Modeling the complex deformations of cylindrical tubes under external pressure is of interest in engineering and physiological applications. The highly non linear post buckling behavior of cross section of the tube during collapse attracted researchers for years. Major efforts were concentrated on studying the behavior of thin wall tubes. Unfortunately, the knowledge on post buckling of thick wall tubes is still incomplete, although many experimental and several theoretical studies have been performed. In this study we systematically studied the effect of the wall thickness on post buckling behavior of the tube. For this purpose, we utilized a computational model for evaluation of the real geometry of the deformed cross sectional area due to negative transmural (internal minus external) pressure. We also developed an experimental method to validate the computational results. Based on the computed cross sections of tubes with different wall thicknesses, we developed a general tube law that accounts for thin or thick wall tubes and fits the numerical data of computed cross sectional areas versus transmural pressures.
\end{abstract}

Tube-law, Collapsible elastic tubes, Thick-wall tubes, Ultrasound imaging, Active contours

\section{Introduction}

Modeling complex deformations of cylindrical tubes under external pressure is of interest in engineering and physiological applications. One of the applications is fluid flow through com pliant tubes, which usually represents physiological flows such as blood flow in arteries or air flow in the airways. This problem is known as fluid structure interaction (FSI) since the fluid and the structure (i.e., tube wall) are continuously in contact and at dynamic equilibrium. The flowing fluid is governed by the con tinuity and momentum equations and the motion of the tube wall is governed by dynamic equilibrium and constitutive equa tions. Fluid flow through a compliant conduit represents a three dimensional problem which is complicated for analytical solutions and requires advanced numerical solutions. This complexity has been overcome by solving simplified one dimensional (1D) mod els of fluid flow through collapsible tubes. In 1D models, the equilibrium and constitutive equations that govern the tube wall motion are replaced by a tube law that relates the transmural pressure $P_{t m}=P_{i} \quad P_{e}$ (i.e., $P_{i}$ is the internal pressure and $P_{e}$ the external pressure) to the corresponding cross sectional area of the tube (Shapiro, 1977).
The pressure area curve of the tube law of compliant cylind rical conduits exhibits two distinct regions. For positive transmural pressures, the cross section inflates and becomes circular, the walls are stretched and the perimeter increases. For negative transmural pressures, the tube buckles and the cross section flattens until the opposite walls come into contact. While the inflation of the tube can be easily solved analytically, the highly non linear post buckling behavior requires more complex theore tical and numerical solutions, and thus, attracted researches for years. Major efforts were focused on studying the post buckling behavior of thin wall tubes and models were developed using the thin shell theory (Yamaki, 1969; Flaherty et al., 1972; Heil and Pedley, 1996; Kounadis, 2006; Whittaker et al., 2010). Several attempts were made to fit the classical thin shell theories for studying the post buckling deformation of thick wall tubes by adding correction factors (Simitses, 1996; Chroscielewski et al., 2010). These efforts led to more accurate results for tubes with moderate wall thickness, but failed to properly describe the post buckling behavior of tubes with very thick walls. Several experi mental works (Bertram, 1982, 1987; Bertram et al., 1990; Bertram and Elliott, 2003) and numerical studies (Marzo et al., 2005; Zhu et al., 2008, 2010, 2013) were performed to study the post buckling behavior of thick wall tubes, but for limited values of wall thickness.

Computational investigations of 1D models of fluid flow in collapsible tubes require analytical formulations of the tube law 
with continuity between the collapse and inflated regions. How ever, a good fit to experimental data requires complex formula tions with many coefficients (Bassez et al., 2001). Several simple analytic relationships were introduced for the tube law of com pliant tubes, but they did not account for the full mechanical contribution of wall thickness on the dimensionless pressure area curves (Shapiro, 1977; Elad et al., 1987; Ottesen, 2003).

In this study we explored the role of wall thickness in post buckling behavior of collapsible tubes. We utilized a computa tional model for evaluation of the accurate geometry of the tube deformed cross sectional area due to negative transmural pres sure. We also developed an experimental method to validate the computational results. Based on the computed cross sections of tubes with different wall thicknesses, we developed a general tube law for both thin and thick wall elastic tubes.

\section{Methods}

\subsection{Physical model}

The physical model of this work was a very long tube, $L \gg R_{i}$, with $\gamma h / R_{i}$, where $L$ was the tube length, $R_{i}$ the internal radius and $h$ the wall thickness. The tube cross-section was assumed to be circular and unstressed for $P_{t m} 0$ (Fig. 1). In this work we investigated the 2-fold symmetric collapse of thin- and thickwall tubes when subjected to $P_{t m}<0$.

\subsection{Computational model}

The computational analysis of the post-buckling geometry of the tube crosssectional areas was conducted for two-dimensional (2D) models of the collapsed cross-section for thin- and thick-wall tubes. Since the tube was relatively long (i.e., $L \gg R_{i}$ ), it was reasonable to neglect axial strains and the problem was reduced to plain strain analysis. The 2D model was formulated for an unstressed circular crosssection at $P_{t m} \quad 0$ (Fig. 1). The deformation of the tube cross-section for a given $P_{t m}<0$ is a static problem, and thus, the solid domain of the tube wall deformation was governed by

KU $\mathbf{F}$

where $K$ is the stiffness matrix, $\mathrm{U}$ is the displacement vector, and $\mathrm{F}$ is the vector of external loads.

Similar to Marzo et al. (2005), convergence problems were observed for tubes with wall thickness ratios of $\gamma \geq 0.333$. Hence we applied the "low speed dynamics" analysis by implementing a damping term in Eq. (1) (ADINA, 2011). Thus, the solid domain for very thick-wall tubes (i.e., $\gamma \geq 0.333$ ) was governed by

$$
\beta K \dot{\mathbf{U}}+K \mathbf{U} \quad \mathbf{F}
$$

where $\dot{\mathbf{U}}$ is the velocity vector and the coefficient $\beta>0$ is an empirical damping parameter. tions:

The model was fully constrained by defining the following boundary condi-

$\mathbf{U}_{x}\left(\begin{array}{lll}x & 0, y) & 0\end{array}\right.$

$\mathbf{U}_{y}(x, y \quad 0) \quad 0$

where $\mathbf{U}_{x}$ and $\mathbf{U}_{y}$ are the $x$ and $y$ components of the displacement vector. In practice, lines $A B$ and $C D$ could move only in the $x$-direction, while line $E H$ could move only in the $y$-direction (Fig. 1).

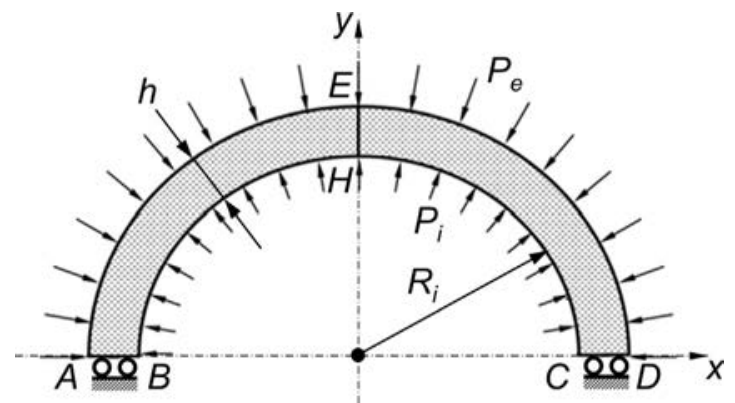

Fig. 1. Schematic model of the circular unstressed collapsible tube.
The tube wall may undergo large displacements and strains, and thus, it was further assumed to behave as a Neo-Hookean hyperelastic material for which the strain energy density function is given by

$W \quad \frac{G}{2} \cdot\left(\lambda_{1}^{2}+\lambda_{2}^{2}+\lambda_{3}^{2}-3\right)+\frac{\kappa}{2}\left(\lambda_{1} \lambda_{2} \lambda_{3}-1\right)^{2}$

where $\lambda$ is the stretch ratio, $G \quad E / 2(1+\nu)$ is the shear modulus, $\kappa \quad E / 3(1-2 \nu)$ is the bulk modulus, $E$ is the Young's modulus and $\nu$ is the Poisson's ratio of the tube.

The model was discretized with 9-node quadrilateral elements and solved with ADINA finite-element software package (version 8.8, Watertown, NY, USA). The external pressure, $P_{e}$, was incrementally applied in thousands of load steps to the lateral surface of the tube, while the internal pressure was kept at $P_{i} \quad 0$. Applying a uniform external pressure on the unstressed circular cross-section of the tube did not yield the 2-fold collapse mode of buckling, but resulted in decrease of the diameter and led to non-convergence of the numerical solutions. To overcome this problem we imposed an ad-hoc initial geometry perturbation and assumed a slightly elliptical initial cross-section of the tube with a semi-major axis of $1.001 R_{i}$ and semi-minor of $0.999 R_{i}$

We conducted the analysis for tubes with $R_{i} 9 \mathrm{~mm}, h \quad 0.09,0.9,2,3$ and $4.5 \mathrm{~mm}$ (i.e., $\gamma \quad 0.01,0.1,0.222,0.333$ and 0.5 ), $E \quad 3.43 \mathrm{MPa}$ and $\nu \quad 0.4$. Since the cross-section of the tube demonstrated strongly unstable behavior on the onset of the buckling, we carefully performed comprehensive convergence tests to explore the optimal mesh density and number of load steps in which the external pressure was applied. For each transmural pressure, the cross-sectional area was calculated and compared to the cross-sectional area obtained from the finer solution (i.e., larger number of elements or load steps). Such tests were performed for each simulated thickness ratio, $\gamma$. For tubes with $\gamma<0.5$ a mesh of 500 elements was found to give the solution with a maximal difference of $\sim 0.2 \%$ with respect to finer mesh. For the tube with $\gamma \quad 0.5$, a mesh of 3600 elements was needed to give the accuracy of $3.4 \%$ in the calculated cross-sections. A number of 10,000 load steps for a thin-wall tube $(\gamma \quad 0.01)$ and 50,000 load steps for the thicker-wall tubes $(\gamma \geq 0.1)$ resulted in cross-sections with maximal differences of $1.4 \%$ and $0.1 \%$ compared to the finer case, respectively. A damping parameter of $\beta 1 \times 10^{6}$ was used for tubes with $\gamma \geq 0.333$ in order to ensure numerical convergence. Sensitivity tests showed that $\beta \quad 1 \times 10^{6}$ resulted cross-sections with maximal difference of $0.21 \%$ with respect to the steady state analysis (i.e., $\beta 0$ ).

\subsection{Experimental investigation}

\subsubsection{Experimental system}

Commercial tubes made of Silicone rubber were used to explore the contours of post-buckling deformation of the cross-section of thick-wall tubes with different $\gamma$ when subjected to $P_{t m}<0$. The water-filled tube was mounted horizontally between coaxial supports in a water-filled tank (Fig. 2). One end of the tube was connected to a vacuum pump via a water reservoir, while the other end was connected to a pressure transducer. The pressure transducer (143PC05D, Omega Engineering, USA; accuracy of $\pm 1.5 \mathrm{~mm} \mathrm{Hg}$ ) was connected to a laptop equipped with an analog-to-digital card. The tube length was at least 10 times longer than the outer diameter. A system for B-mode ultrasound (US) imaging (Voluson E6, GE, USA) was used to acquire the geometry of the collapsed cross-sections at the middle of the tube and far away from the ends. The US transducer was held with a custom designed fixture perpendicular to the tube axis. Acquisition of the US image and $P_{t m}$ was done after stabilization of the tube geometry at the given pressure.

\subsubsection{Image processing of US images}

The recorded US images were converted to BMP files for image processing and data analysis using Matlab. The region of interest was selected for processing as shown in Fig. 3a. For identification of the contour of the inner cross-section of the tube we utilized the Vector Field Convolution (VFC) method of the active contour model using the Active Model Toolbox of Matlab (Li and Acton, 2007, 2008). This method required manual initialization of the expected contour by several points and conversion of the image into a binary image using a threshold algorithm. Visual inspection of a US image revealed a clear bright band on both sides of the tube wall at the interface between the tube wall (i.e., Silicone rubber) and the water (Fig. 3a). Based on ultrasonography experience in obstetrics, the inner wall-fluid interface is expected to be outside of the bright band for the inner cross-section of the tube (Salomon et al., 2010). Accordingly, we first marked 8-10 points on the image on the expected contour of the collapsed cross-section (Fig. 3b). These points were connected with straight lines to generate the initial iteration for the contour vector of about 200 points. The missing points on the straight lines were computed with an active contour re-mesh routine. In order to yield the binary image, the $30 \%$ brightest pixels in circles of 10 pixels radius around each of the vector points were detected and the remaining black pixels inside the detected band were converted to white pixels (Fig. 3c). Application of the VFC algorithm provided smooth contour for the inner cross-section within 40 iterations (Figs. 3d and e). Finally, the inner cross-sectional area of the collapsed tube was calculated by integration of the detected contour 


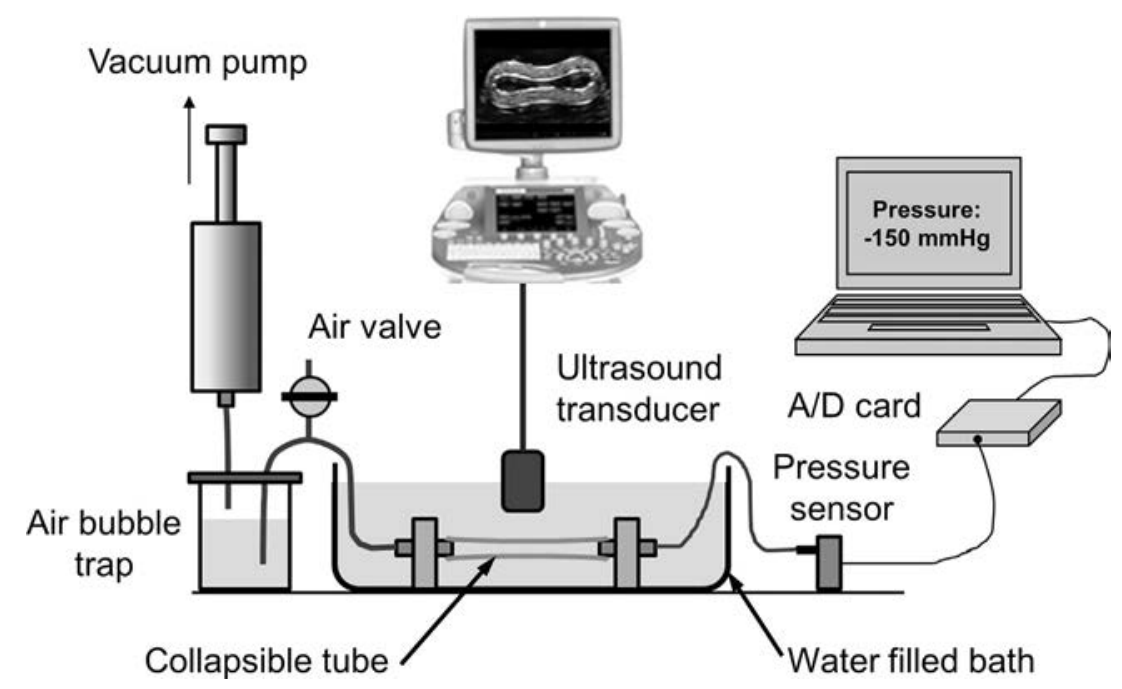

Fig. 2. Experimental setup for imaging the collapsed cross-sections of thick-wall elastic tubes.
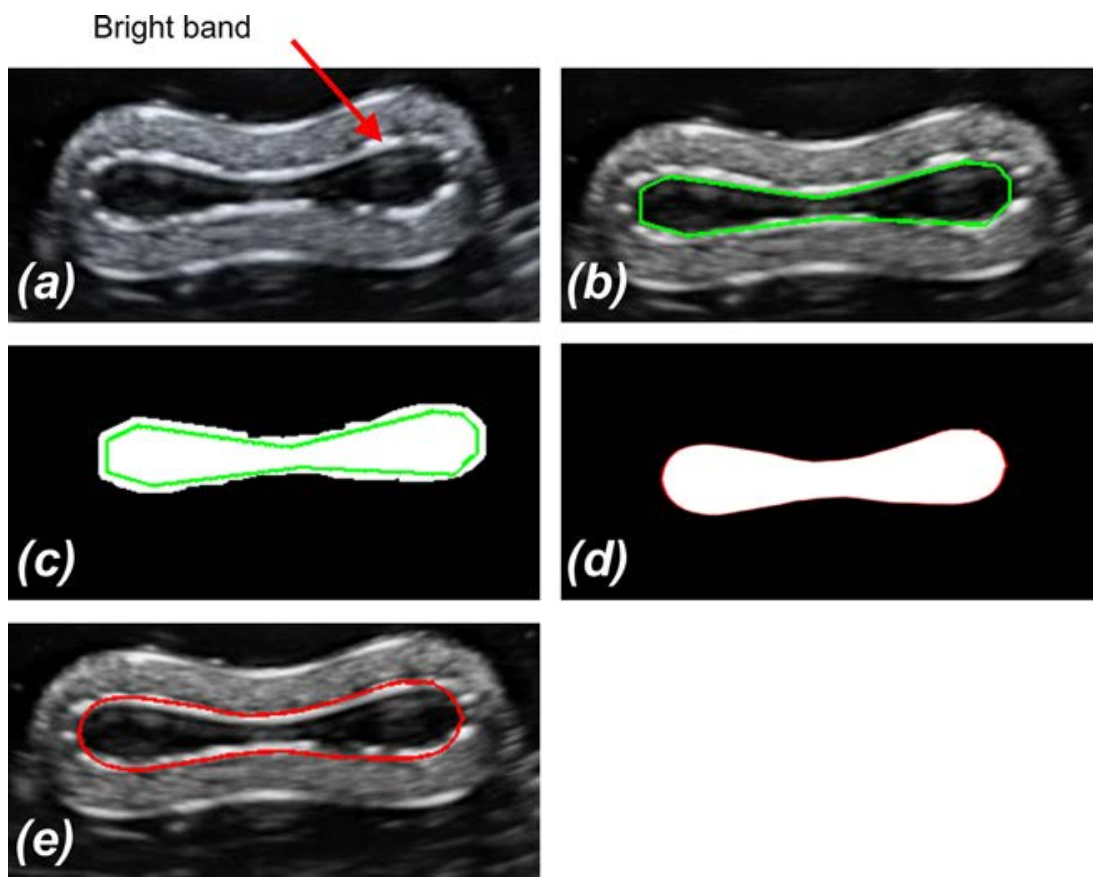

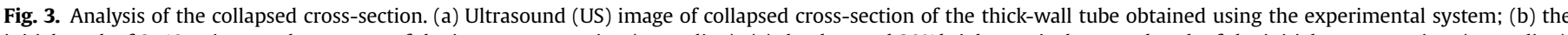

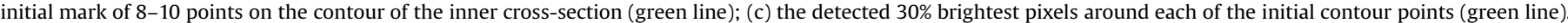

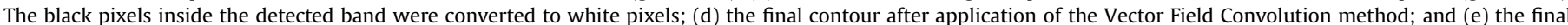
contour displayed on the original US image. (For interpretation of the references to color in this figure legend, the reader is referred to the web version of this article.)

\subsection{Validation of computational model}

\subsubsection{Thin-wall tubes}

The contours of the collapsed cross-sections for a thin-wall elastic tube were computed both with ADINA (as described in Section 2.2) and the analytic model of Flaherty et al. (1972). For the computation with ADINA we assumed a tube with $R_{i} \quad 9 \mathrm{~mm}, h \quad 0.09 \mathrm{~mm}$ (i.e., $\gamma \quad 0.01$ ), E $\quad 3.43 \mathrm{MPa}$ and $\nu \quad 0.4$. The boundary value problem of the analytic model is governed by a system of six ordinary differential equations and boundary conditions (for details see Flaherty et al. (1972)), and was solved using MATLAB bvp4c subroutine.

\subsubsection{Thick-wall tubes}

The computed contours of the cross-sectional areas of thick-wall tubes using ADINA were validated by comparison to the experimental cross-sections obtained from the analysis of US images. We measured the collapsed geometry of 2 tubes; one with $\gamma \quad 0.24$ (e.g., $R_{i} \quad 12.5 \mathrm{~mm}, h \quad 3 \mathrm{~mm}, E \quad 2.44 \mathrm{MPa}, \nu \quad 0.4$ ) and a thicker one with $\gamma \quad 0.333$ (e.g., $R_{i} \quad 9 \mathrm{~mm}, h \quad 3 \mathrm{~mm}, E \quad 4.0 \mathrm{MPa}, \nu \quad 0.4$ ). Circumferential strips of the tested tubes were stretched in an Instron 5544 system in order to evaluate their Young's modulus for the computational analysis. The tubes were subjected to negative transmural pressures and the cross-sections were detected using the VFC algorithm.

\subsection{Normalization of the results}

The results were presented using non-dimensional variables in order to allow comparison with the theoretical solution by Flaherty et al. (1972), as well as comparison between tubes with different ratios $\gamma$. The transmural pressure was scaled by the flexural rigidity of the tube $K_{p}$ (for details see Appendix A)

$\Pi \frac{P_{t m}}{K_{p}}$ 
$K_{p} \frac{E}{12\left(1-\nu^{2}\right)}[\ln (1+\gamma)]^{3}$

The cross-sectional area of the tube, $A$, was scaled by the undeformed crosssectional area, $A_{0}$,

$\alpha \frac{A}{A_{0}}$

The $x$ and $y$ coordinates of the physical domain were scaled by the internal radius of the tube

$\xi \frac{x}{R_{i}} \quad \eta \frac{y}{R_{i}}$

\section{Results}

In this work we explored the effect of wall thickness on the behavior of elastic tubes during collapse. Accordingly, we investi gated the whole range of wall thickness from very thin to very thick walls; namely, $\gamma=0.01,0.1,0.222,0.333$ and 0.5 . All the tubes demonstrated similar patterns of deformation during collapse, as shown in Fig. 4. When the transmural pressure became negative, the tube cross section underwent small axisymmetric pre buckling deformations, the perimeter slightly decreased and wall thickness slightly increased. As the negative transmural pressure exceeded the buckling pressure $\Pi_{b}$, the cross sectional area rapidly decreased; it first became elliptical and then flattened until the opposite walls came into contact point for which the transmural pressure is $\Pi_{c p}$.

\subsection{Validation of numerical solution}

\subsubsection{Thin wall tubes}

The computed pressure area curve for a thin wall tube (i.e., $\gamma=0.01$ ) perfectly fitted the results of the classical model of Flaherty et al. (1972) for a wide range of transmural pressures (Fig. 5a). A maximal difference of $0.72 \%$ was observed on the onset of buckling at $\Pi_{b}=3$ (Fig. $5 \mathrm{a}$ ). The calculated contours of the cross sections were also identical to those predicted by the analytical solution (Figs. 5b d).

\subsubsection{Thick wall tubes}

The collapsed cross sections of the thinner thick wall tube (e.g., $\gamma=0.24$ ) were acquired via US imaging for several values of transmural pressures and later analyzed to provide the relevant contours and cross sectional areas. The contours of the cross sections obtained from the processing of US images matched the

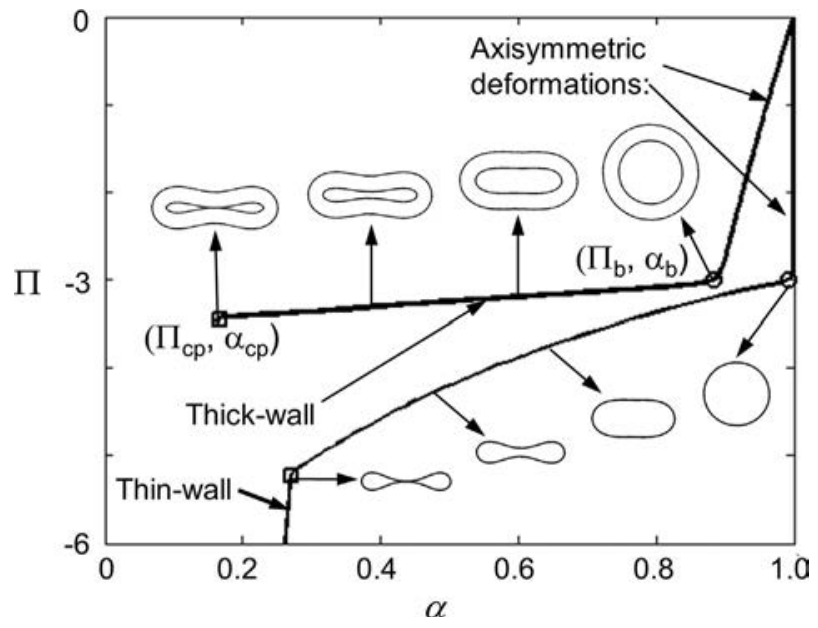

Fig. 4. Typical non-dimensional pressure-area curves for thin (thin line) and thick (thick line) wall collapsible tubes. Buckling point (circle), contact point (rectangle) and collapsed cross-sections are marked on the curves. computed cross section (Figs. $6 \mathrm{~b}$ d). The experimental data fitted well with the pressure area curve obtained from the numerical simulations, with maximal deviations of $5.22 \%$, which is within the range of accuracy of the pressure transducer (Fig. 6a). The computational results for the thicker thick wall tube (e.g., $\gamma=0.333$ ) were also in good agreement with experimental data with maximal differences of $4.35 \%$ (Fig. 7).

\subsection{The effect of thickness ratio on behavior of collapsible tubes}

The computational results for all the tubes were summarized as pressure area curves as shown in Fig. 8. An interesting outcome is that all the tubes buckled at the same transmural pressure, $\Pi_{b}=3$. This single point of buckling in the region of the smooth knee at the onset of buckling was found by extrapolating the almost straight segments on both sides until they met. As the tube wall was thicker, it underwent larger axisymmetric pre buckling deformations and buckling occurred at smaller cross sections $\alpha_{b}$ (Fig. 8). Throughout the axisymmetric pre buckling deformations, the perimeter of the cross section decreased by $0.051 .5 \%$ and the wall thickness increased by $0.0012 .8 \%$ from its initial values for thinner $(\gamma=0.01)$ and thicker $(\gamma=0.5)$ tubes, respectively. During the transit from buckling to opposite wall contact point $\left(\alpha_{c p}\right)$, the slope of the pressure area curve (i.e., $d \Pi / d \alpha$ ) decreased as wall thickness of the tube increased. Accordingly, the contact point pressure $\Pi_{c p}$ was less negative and the cross sectional area $\alpha_{c p}$ was smaller for tubes with thicker walls.

\subsection{Development of the general tube law}

Based on the computed cross sections, which are given in Fig. 8, we assumed the following general tube law similar to Elad et al. (1987) for each of the computed tubes

$\Pi(\alpha, \gamma)=c_{1}(\gamma) \cdot\left(\alpha^{m_{1}(\gamma)} \quad a^{m_{2}(\gamma)}\right)$

where $c_{1}(\gamma), m_{1}(\gamma)$ and $m_{2}(\gamma)$ are tube wall coefficients, which were assumed to be functions of the wall thickness ratio $\gamma$. Using a non linear least square fitting procedure, the coefficients were found for each tube for the range $\alpha_{c p}(\gamma) \leq \alpha \leq 1$. These coefficients were then expressed as functions of the wall thickness ratio $\gamma$ as follows:

$c_{1}=3.1=$ const $\quad m_{1}(\gamma)=\frac{60}{\gamma^{0.5}} \quad 65 \quad m_{2}(\gamma)=0.7 \gamma \quad 0.4$

Substituting Eq. (11) into Eq. (10) yielded a general tube law that also counts for elastic tubes with either thin or thick walls,

$\Pi(\alpha, \gamma)=3.1\left(\alpha^{60 / \gamma^{05}-65} \quad \alpha^{0.7 \gamma-0.4}\right), \quad \alpha_{c p}(\gamma) \leq \alpha \leq 1$

The general tube law for different values of $\gamma$ is plotted in color solid lines together with computational results in color circles as shown in Fig. 9.

\section{Discussion}

In the present study we have investigated the collapse char acteristics of thin and thick wall elastic tubes subjected to negative transmural pressures. The computed data was generated using the commercial finite element software of ADINA, whereas the experi mental data was obtained via US imaging. The effects of wall thickness on the post buckling behavior of the tubes were thor oughly studied for a wide range of wall thickness values. A general tube law that accounts for thin and thick wall tubes was developed to fit the numerical data of the computed cross sectional areas versus transmural pressures. 
a

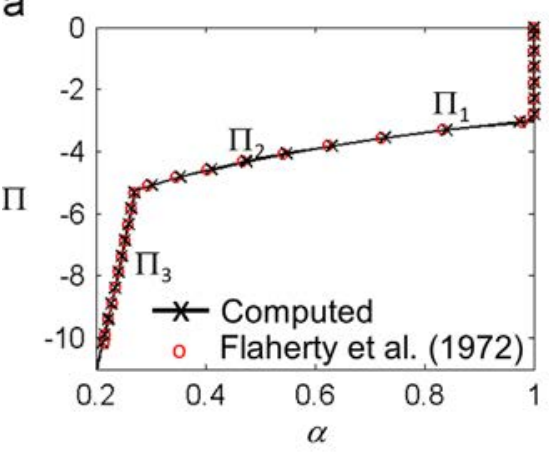

C

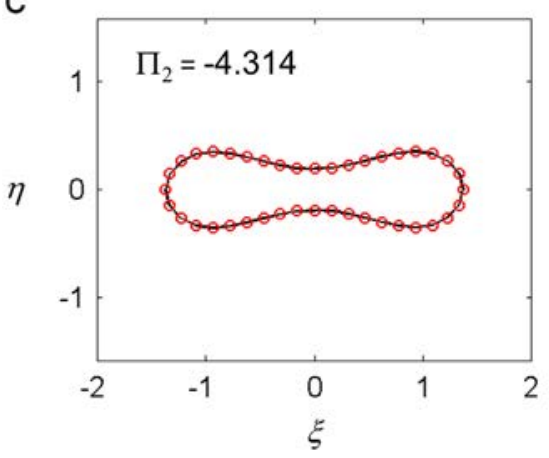

b

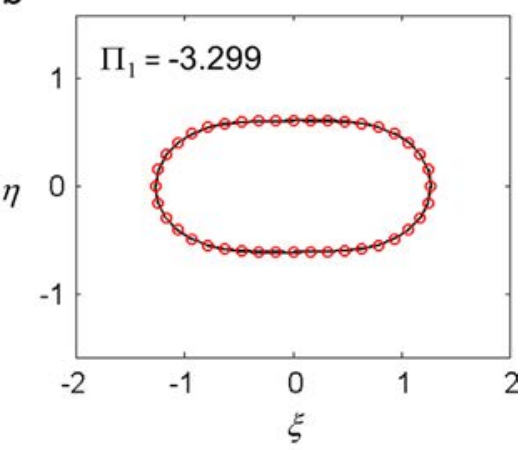

d

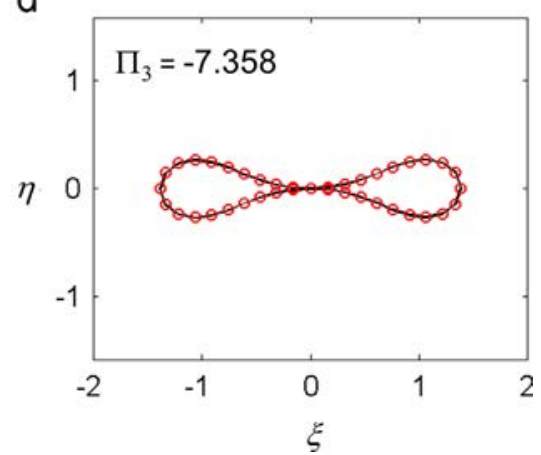

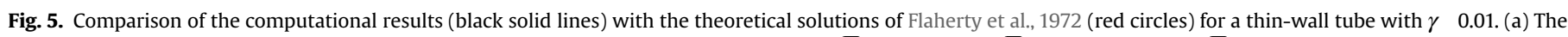

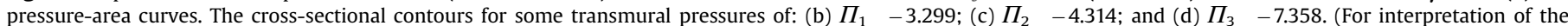
references to color in this figure legend, the reader is referred to the web version of this article.)

a

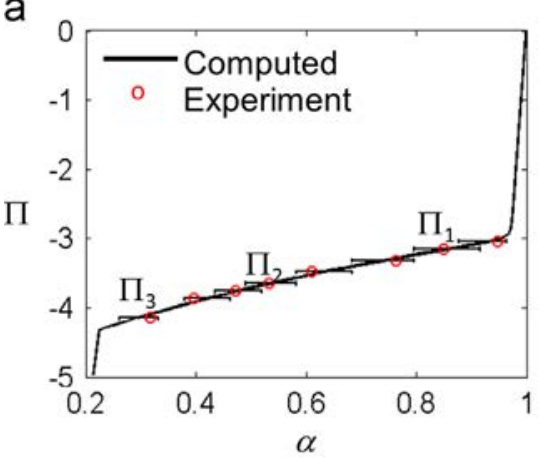

C

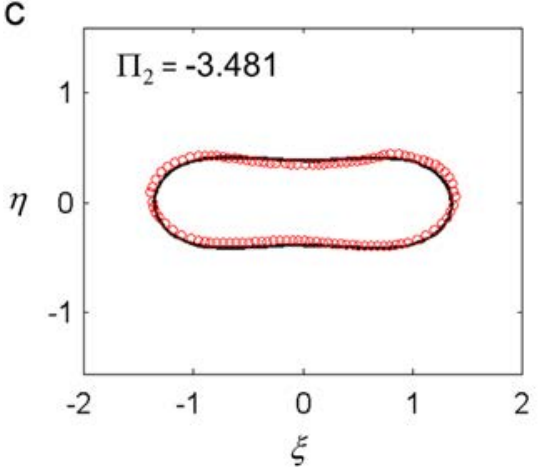

b

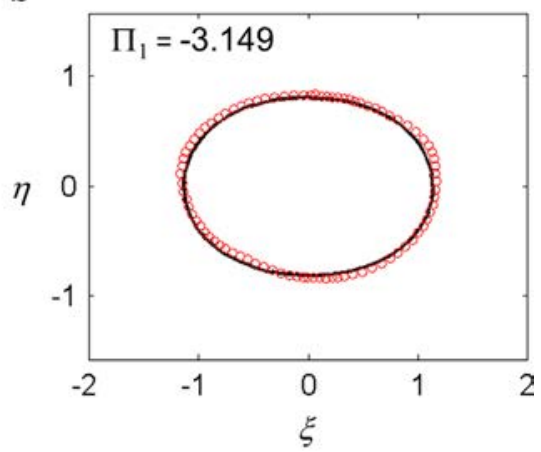

d

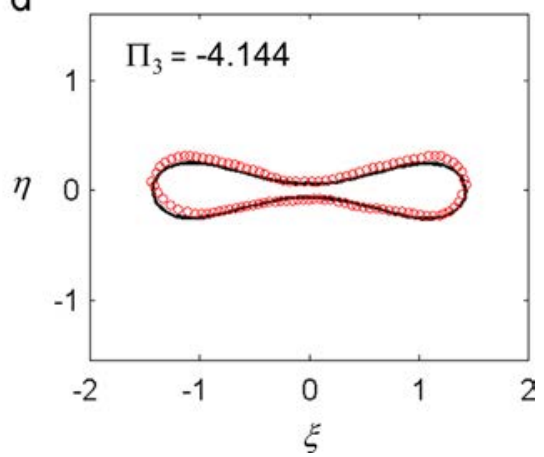

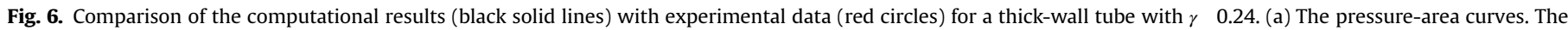

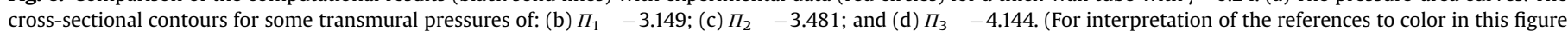
legend, the reader is referred to the web version of this article.)

The validation of numerical scheme is an important phase in developing a computational model. Due to strongly unstable behavior of elastic tubes during collapse, we paid special attention to numerical stability tests such as the number of mesh elements and load steps. We also carefully examined the validation of the numerical predictions comparing the computational results with 

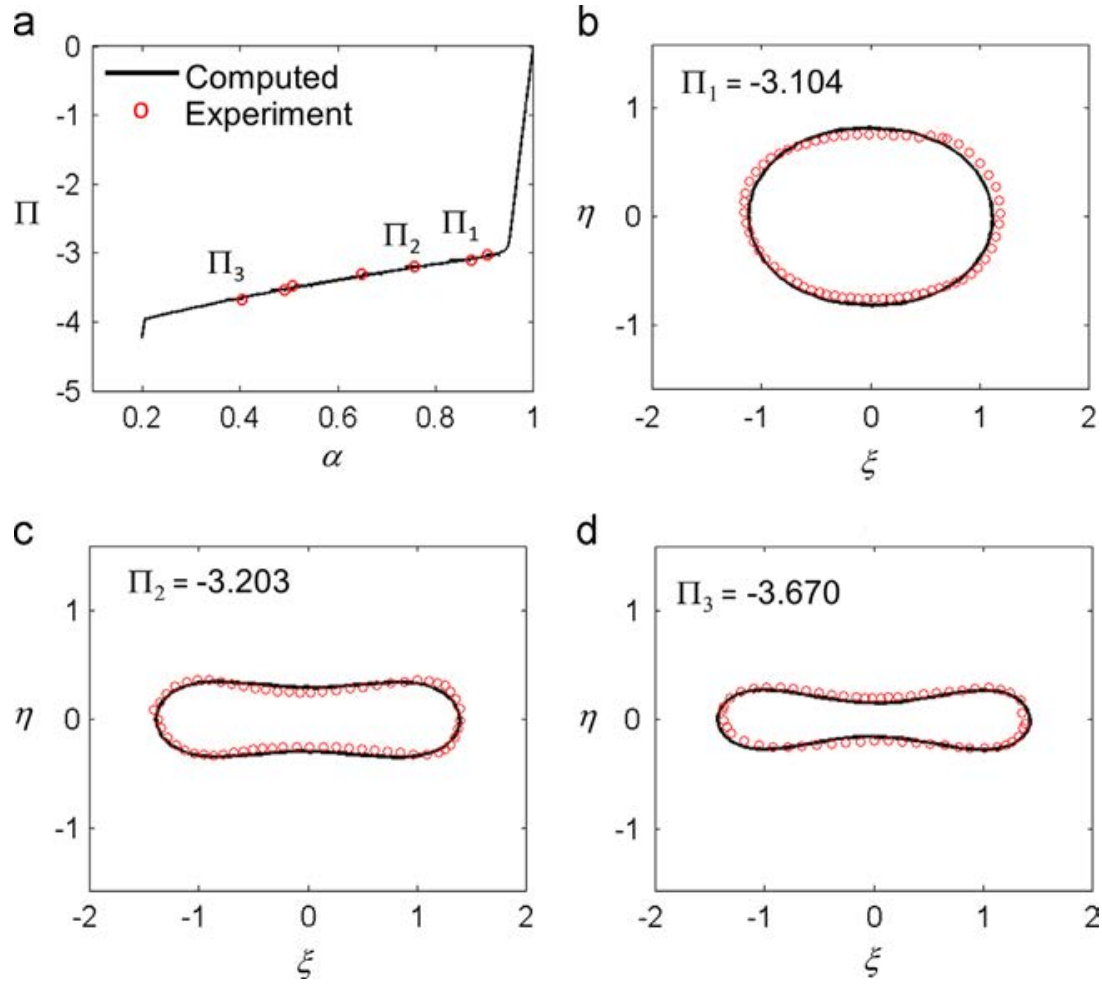

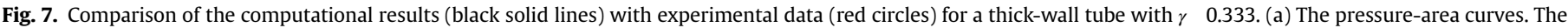
cross-sectional contours for some transmural pressures of: (b) $\Pi_{1} \quad-3.104$; (c) $\Pi_{2} \quad-3.203$; and (d) $\Pi_{3} \quad-3.670$.

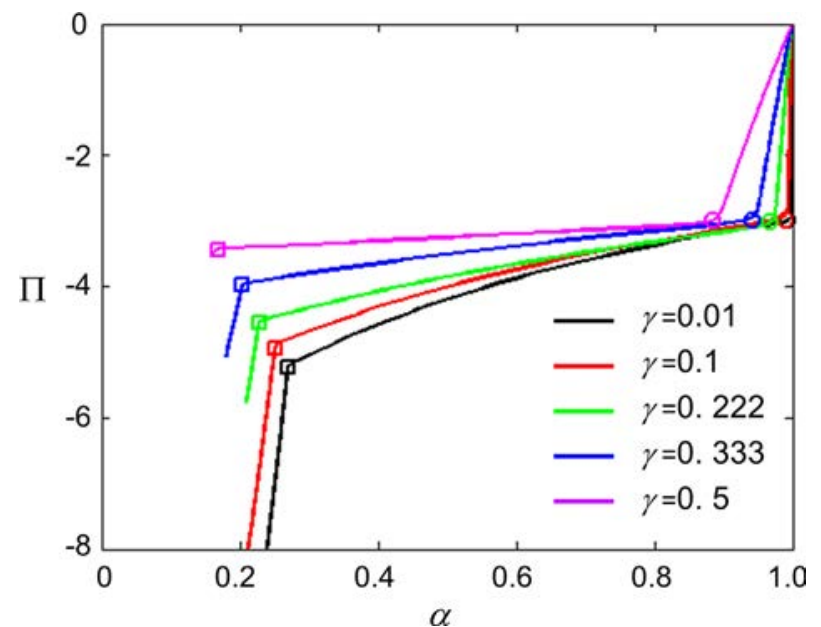

Fig. 8. Non-dimensional pressure-area curves for tubes with different $\gamma$ computed from the data obtained from ADINA simulations. Symbols were added on each curve to mark the buckling points with "circles" and the contact points with "rectangles".

classical theoretical solutions for thin wall tubes (Flaherty et al., 1972) and to experimental data for thick wall tubes. We demon strated very good agreement of the computational results with the theoretical and experimental data for both thin and thick walls. Previous attempts to compare computational and experi mental results for thick wall collapsible tubes yielded much greater differences (Marzo et al., 2005).

Comprehensive evaluation of the effect of wall thickness on post buckling behavior of tubes revealed several behavior patterns. First, before buckling occurred, the tubes underwent axisymmetric deformation, which was previously observed (Heil and Pedley, 1995). The flexural rigidity, $K_{p}$, increases with the wall thickness to radius ratio $\gamma$ (Eq. 7), and thus, the resistance of thicker tubes to bending is much greater. Hence, the axisymmetric

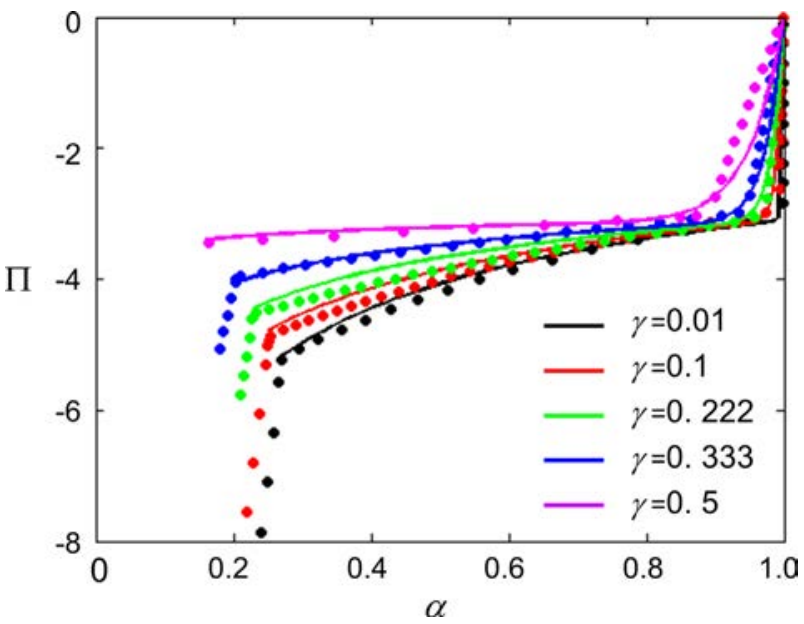

Fig. 9. Non-dimensional pressure-area curves as obtained from ADINA simulations (circles), and the general tube law given by Eq. (12) (solid lines) for tubes with different $\gamma$.

pre buckling deformations increased for thick wall tubes. Accord ingly, the buckling cross sectional area $\alpha_{b}$ was significantly lower for tubes with thick walls. Nevertheless, all thick wall tubes that were simulated in this study, as well as thin wall tubes, buckled at the same transmural pressure $\Pi_{b}=3$, as previously demon strated (Weissman and Mockros, 1967; Bertram, 1987). Once buckling occurred, thick wall tubes were more compliant than thin wall tubes (Bertram, 1987; Marzo et al., 2005; Zhu et al., 2013), and accordingly, larger reductions of the cross sectional area occurred at less negative transmural pressures.

The collapse patterns of thick wall elastic tubes found in this work have already been demonstrated in previous studies, but for a limited number of wall thicknesses (Bertram, 1987; Marzo et al., 2005; Zhu et al., 2013). The effects of wall thickness to radius and 
length to radius ratios on obtaining different theoretical long itudinal and axial modes of buckling in thin and thick wall tubes were systematically studied (Zhu et al., 2008). However, the effects of wall thickness on the pressure area curve were not clearly demonstrated. In our modeling, we used a validated computa tional model to demonstrate the pattern of post buckling defor mation of elastic tubes for a wide and continuous range of wall thicknesses. We successfully modeled the post buckling behavior including the point contact and line contact conditions, which were not conducted in previous computational studies (Marzo et al., 2005; Zhu et al., 2008, 2010, 2013).

The simplified tube laws that have been implemented in previ ous studies of 1D fluid flow in compliant tubes did not describe the physical pressure area curve (Shapiro, 1977; Elad et al., 1987; Ottesen, 2003; Bringley et al., 2008). Specifically, the buckling itself was ignored, since large deformations occurred even for negative transmural pressures while $3<\Pi<0$. Moreover, none of the published models that describe the pressure area curves of compliant tubes considered the effect of wall thickness ratio and hence they can at most describe the post buckling behavior of a specific tube with single value of $\gamma$.

The major outcome of this study is the new general tube law that includes the physical contribution of wall thickness for description of the pressure area relationship for a wide range of thickness to radius ratios. The relatively simple analytical formu lation of the tube law (Eq. 12) is very important for utilization in 1D models of fluid flow in compliant tubes. Application of this tube law will allow more accurate modeling of fluid flow through thick wall compliant tubes with longitudinally varying geometri cal and mechanical properties, which are naturally presented in physiological conduits.

\section{References}

ADINA, R.D., 2011. ADINA: A theory and modeling guide. Adina R\&D, Inc., Watertown, MA.

Bassez, S., Flaud, P., Chauveau, M., 2001. Modeling of the deformation of flexible tubes using a single law: application to veins of the lower limb in man.J. Biomech. Eng. 123, 58-65.
Bertram, C.D., 1982. Two modes of instability in a thick-walled collapsible tube conveying a flow. J. Biomech. 15, 223-224.

Bertram, C.D., 1987. The effects of wall thickness, axial strain and end proximity on the pressure-area relation of collapsible tubes. J. Biomech. 20, 863-876.

Bertram, C.D., Elliott, N.S.J., 2003. Flow-rate limitation in a uniform thin-walled collapsible tube, with comparison to a uniform thick-walled tube and a tube of tapering thickness. J. Fluids Struct. 17, 541-559.

Bertram, C.D., Raymond, C.J., Pedley, T.J., 1990. Mapping of instabilities for flow through collapsed tubes of differing length. J. Fluids Struct. 4, 125-153.

Bringley, T.T, Childress, S., Vandenberghe, N., Zhang, J., 2008. An experimental investigation and a simple model of a valveless pump. Phys. Fluids $20(033602-$ $1-15)$.

Chroscielewski, J., Pietraszkiewicz, W., Witkovski, W., 2010. On shear correction factors in the non-linear theory of elastic shells. Int. J. Solids Struct. 47, 3537-3545.

Elad, D., Kamm, R.D., Shapiro, A.H., 1987. Choking phenomena in a lung-like model J. Biomech. Eng. 111, 109-199.

Flaherty, J.E., Keller, J.B., Rubinow, S.I., 1972. Post buckling behavior of elastic tubes and rings with opposite sides in contact. SIAM J. Appl. Math. 23, 446-455.

Heil, M., Pedley, T.J., 1995. Large axisymmetric deformation of a cylindrical shell conveying a viscous flow. J. Fluids Struct. 9, 237-256.

Heil, M., Pedley, T.J., 1996. Large post-buckling deformations of cylindrical shells conveying viscous flow. J. Fluid Struct. 10, 565-699.

Kounadis, A.N., 2006. Recent advances on postbuckling analyses of thin-walled structures: beams, frames and cylindrical shells. J. Constr.Steel Res. 62, 1101-1115

Li, B., Acton, S.T., 2007. Active contour external force using vector field convolution for image segmentation. IEEE Trans. Image Process. 16, 2096-2106.

Li, B., Acton, S.T., 2008. Automatic active model initialization via Poisson inverse gradient. IEEE Trans. Image Process. 17, 1406-1420.

Marzo, A., Luo, X.Y., Bertram, C.D., 2005. Three-dimensional collapse and steady flow in thick-walled flexible tubes. J. Fluids Struct. 20, 817-835.

Ottesen, J.T., 2003. Valveless pumping in a fluid-filled closed elastic tube-system: one-dimensional theory with experimental validation. J. Math. Biol. 46, 309-332.

Salomon, L.J., Alfirevic, Z., Berghella, V., Bilardo, G., Hernandez-Andrade, E., Jonsen, S.L., Kalache, K., Leung, K.Y., Malinger, G., Munoz, H., Prefumo, F., Toi, A., Lee, W. 2010. Practice guidelines for performance of the routine mod-trimester fetal ultrasound scan. Ultrasound Obstet. Gynecol. 41, 348-359.

Shapiro, A.H., 1977. Steady flow in collapsible tubes. J. Biomech. Eng., 126-147.

Simitses, G.J., 1996. Buckling of moderately thick laminated cylindrical shells: a review. Compos. B: Eng. 27, 581-587.

Weissman, M., Mockros, L., 1967. The mechanics of a collapsing tube heart pump. Int. J. Mech. Sci. 9, 113-121.

Whittaker, R.J, Heil, M., Jensen, O.E., Waters, S.L., 2010. A rational derivation of a tube law from shell theory. Q. J. Mech. Appl. Math. 63, 465-496.

Yamaki, N., 1969. Buckling of circular cylindrical shells under external pressure. Rep. Inst. High Speed Mech. 20, 35-55.

Zhu, Y., Luo, X.Y., Ogden, R.W., 2008. Asymmetric bifurcations of thick-walled circular cylindrical elastic tubes under axial loading and external pressure. Int. J. Solids Struct. 45, 3410-3429.

Zhu, Y., Luo, X.Y., Ogden, R.W., 2010. Nonlinear axisymmetric deformations of an elastic tube under external pressure. Eur. J. Mech.: A/Solids 29, 216-229.

Zhu, Y., Luo, X.Y., Wang, H.M., Ogden, R.W., Berry, C., 2013. Three-dimensional nonlinear buckling of thick-walled elastic tubes under pressure. Int. J. Non-Linear Mech. 48, 1-14. 